\title{
Reflections on the residual finiteness of one-relator groups
}

\author{
Gilbert Baumslag, Charles F. Miller III and Douglas Troeger
}

\begin{abstract}
Let $G=\langle a, b, \ldots \mid r=1\rangle$ be a one-relator group equipped with at least two generators. For all $w$ which do not commute with $r$ in the ambient free group on the generators $a, b, \ldots$, the groups $G(r, w)=\left\langle a, b, \ldots \mid r^{r^{w}}=r^{2}\right\rangle$ are not residually finite and have the same finite images as $G$. The existence of this family of one-relator groups which are not residually finite reinforces what is becoming more obvious with time, that one-relator groups can be extremely complicated. This not only serves to underline the complexity of one-relator groups but provides us with the opportunity to raise a number of problems about these groups in the hope that they will stimulate further work on the conjugacy and isomorphism problems for one-relator groups as a whole.
\end{abstract}

Mathematics Subject Classification (2000). 20F05, 20E26, 20F10.

Keywords. One-relator group, residually finite, isomorphism problem.

\section{One-relator groups which are not residually finite}

1.1. The main theorem. The purpose of this note is to prove the following theorem.

Theorem 1. Let

$$
G=\langle a, b, \ldots \mid r=1\rangle
$$

be a one-relator group presented on at least two generators. Suppose that $w$ is any element in the ambient free group $F$ on the generators $a, b, \ldots$ which does not commute with $r$, then

$$
G(r, w)=\left\langle a, b, \ldots \mid r^{r^{w}}=r^{2}\right\rangle
$$

is a one-relator group with the same finite images as $G$. Moreover, $r \neq 1$ in $G(r, w)$ and $r$ is contained in every subgroup of finite index in $G(r, w)$. Therefore $G(r, w)$ is not residually finite.

The proof of Theorem 1 depends on the following lemma, which will be proved in Section 3.1. 
Lemma 1. Let $u$ and $v$ be two words in the free group $F$ which do not commute. Then $v^{-1} u v u^{-2}$ is not conjugate in $F$ to either $u$ or $u^{-1}$.

We will record a number of consequences of Theorem 1 in Section 2 and raise a number of open problems about the $G(r, w)$ in Section 4. Here we content ourselves with noting that in the free group $F$ on $a, b$, if $r=a b$ and $w=a$, then $r$ and $a$ do not commute. Hence the group

$$
G(a b, a)=\left\langle a, b \mid(a b)^{(a b)^{a}}=(a b)^{2}\right\rangle
$$

is not residually finite and has the same finite images as the infinite cyclic group $G=\langle a, b \mid a b=1\rangle$. This group was first introduced in [2] with a slightly different presentation.

1.2. Some general remarks about one-relator groups. In $1932 \mathrm{~W}$. Magnus [16] proved that a group defined by a single relation has a solvable word problem. His proof revealed that such groups can be built up from cyclic groups, both infinite and finite, by repeatedly forming amalgamated products in a seemingly straightforward way. This suggests that the conjugacy and isomorphism problems for one-relator groups are also solvable. More than 70 years later, despite many efforts, neither of these problems have been solved.

In 1962, Donald Solitar and the first author proved, in particular, that the groups

$$
B(p, q)=\left\langle a, b \mid a^{-1} b^{p} a=b^{q}\right\rangle,
$$

where $p, q$ are distinct primes, are non-hopfian [6]. So they are not residually finite. The $B(p, q)$ were the first examples of one-relator groups that are not residually finite and provided evidence of the complexity of one-relator groups.

In 1967, the first author proved [1] that if

$$
B(k, \ell, m)=\left\langle a, b \mid\left(a^{-1} b^{k} a b^{\ell}\right)^{m}\right\rangle,
$$

then the group $B(2,3,2)$ is residually finite, although $B(2,3)$ is not. This gave rise to the conjecture that one-relator groups with non-trivial torsion, or as we will refer to them here, one-relator groups with torsion, are all residually finite. In 1968, B. B. Newman [20] proved that one-relator groups with torsion have a solvable conjugacy problem as a consequence of his so-called spelling theorem. This spelling theorem meant that, in today's terminology, one-relator groups with torsion are hyperbolic. Z. Sela [23] has proved that torsion-free hyperbolic groups are hopfian. Now a one-relator group with torsion has a torsion-free subgroup of finite index [14]. One can then deduce, as noted in [4], that one-relator groups with torsion are hopfian.

Prompted by some of the questions left open in [1], Benjamin Baumslag and Frank Levin [5] sketched, in particular, a proof that if $m>1$, then the groups $B(k, \ell, m)$ 
are virtually free by infinite cyclic, i.e., contain a subgroup of finite index which is an extension of a free group by an infinite cyclic group. We have been unable to verify their argument, however. Nevertheless, it is worth noting that since finitely generated virtually free by cyclic groups are residually finite [3], it would follow, as previously observed already in [1], that $B(2,3,2)$ is residually finite. This leads to the possibility that all one-relator groups with torsion are virtually free by cyclic. This remark will be explored in some detail in a paper under development by the authors of this note.

The remarks above serve to indicate that many of the outstanding problems for one-relator groups may well be solvable for one-relator groups with torsion. Indeed S. J. Pride [22] has proved that the 2-generator one-relator groups with torsion have a solvable isomorphism problem. And E. Egerov [8] has proved that if $r$ is a word in the generators $a, b, \ldots, c$ in which no negative powers occur and if $n>1$, then the one-relator group

$$
G=\left\langle a, b, \ldots, c \mid r^{n}=1\right\rangle
$$

is residually finite. Subsequent work by D. Wise [24], [25] and J. McCammond and D. Wise [17], among others, has uncovered hitherto unseen structural properties of one-relator groups with torsion, which suggest that they can well be put together in a new way.

\section{Some corollaries of Theorem 1}

\subsection{Groups whose finite images are abelian}

Corollary 2. Let $r=[a, b]$, let $G=\langle a, b \mid r=1\rangle$ be the free abelian group on $a$ and $b$ and let $w=a$. Then

$$
G(r, a)=\left\langle a, b \mid[a, b]^{[a, b]^{a}}[a, b]^{-2}=1\right\rangle
$$

is not residually finite and all of its finite images are finite abelian groups.

\subsection{Groups whose finite images are metabelian}

Corollary 3. Let $r=b^{a} b^{-2}$ and $w=a$. Then $G=\left\langle a, b \mid b^{a} b^{-2}\right\rangle$ is metabelian and all of the finite images of

$$
G(r, a)=\left\langle a, b \mid\left(b^{a} b^{-2}\right)^{\left(b^{a} b^{-2}\right)^{a}}=\left(b^{a} b^{-2}\right)^{2}\right\rangle
$$

are finite metabelian groups. 


\subsection{Groups with trivial center all of whose non-trivial finite images have centers}

Corollary 4. Let $G(r, a)=\left\langle a, b \mid r^{r^{a}}=r^{2}\right\rangle$ where $r=a^{2} b^{-2}$. Then $G=\langle a, b|$ $r=1\rangle$ has an infinite cyclic center and

(1) $G(r, a)$ has a trivial center;

(2) all of the non-trivial finite factor groups of $G(r, a)$ have a non-trivial center;

(3) $G(r, a)$ has non-abelian finite images;

(4) $G(r, a)$ is not residually finite ( $r \neq 1$ and is contained in every subgroup of finite index).

Since one-relator groups with center are residually finite (see, for example [7]), it follows that $G(r, a)$ is centerless.

2.4. Groups with the same finite images as a free group. Finally we have the following

Corollary 5. Let $G=\langle a, b, \ldots, c \mid r\rangle$, where $r=a b \ldots c$, i.e., is the product of all of the given generators of $G$. Then $G$ is a free group of rank $n-1$ where $n$ is the number of generators of $G$. Hence the n-generator, one-relator group

$$
G(a b \ldots c, a)=\left\langle a, b, \ldots, c \mid(a b \ldots c)^{a}(a b \ldots c)^{-2}\right\rangle
$$

is not residually finite and has the same finite images as the free group of rank $n-1$.

We observe that since free groups are residually finite, $G(a b \ldots c, a)$ is not free.

\section{The proofs of Lemma 1 and Theorem 1}

3.1. The proof of Lemma 1. We denote the length of an element $f$ in a free group by $|f|$.

Proof of Lemma 1. Suppose on the contrary that $v^{-1} u v u^{-2}$ is conjugate in $F$ to $u^{\epsilon}$. Since free groups are residually torsion-free nilpotent, there exists an integer $n$ such that $u \in \gamma_{n}(F)$ and also that $u \notin \gamma_{n+1}(F)$. Then working modulo $\gamma_{n+1}(F)$ we find that

$$
v^{-1} u v u^{-2}=u^{-1}=u^{\epsilon}
$$

which means that $\epsilon=-1$.

The assertion is invariant under inner automorphisms, so we can suppose that $u$ is cyclically reduced. 
Suppose then that for some $w \in F$ we have $v^{-1} u v u^{-2}=w^{-1} u^{-1} w$ or equivalently that

$$
u^{2}=w^{-1} u w v^{-1} u v
$$

We will prove that this is impossible.

Notice that if $v$ and $w$ both end in $u$, i.e., if $v=v_{1} u$ and $w=w_{1} u$ for some $v_{1}$ and $w_{1}$, then $u^{2}=w_{1}^{-1} u w_{1} v_{1}^{-1} u v_{1}$. Since $u$ and $v$ do not commute, neither do $u$ and $v_{1}$. Thus we can assume that $w$ and $v$ do not both end in $u$.

Now $u$ is cyclically reduced. So if we freely reduce the product $w^{-1} u w$ we obtain $w_{0}^{-1} \bar{u} w_{0}$ where $\bar{u}$ is a cyclic permutation of $u, w_{0}$ is a terminal segment of $w$ and where $w_{0}^{-1} \bar{u} w_{0}$ is reduced without cancellation. Similarly, freely reducing $v^{-1} u v$ gives the expression $v_{0}^{-1} \hat{u} v_{0}$ which is reduced without cancellation, where $\hat{u}$ is a cyclic permutation of $u$ and $v_{0}$ is a terminal segment of $v$. This yields the equation

$$
u^{2}=w_{0}^{-1} \bar{u} w_{0} v_{0}^{-1} \hat{u} v_{0}
$$

Since $u$ is cyclically reduced, the left hand side of this equation is reduced as written. On the right-hand side of equation (1) the only place cancellation can occur is at the juncture $w_{0} v_{0}^{-1}$.

In the case when $v_{0}$ is empty, we have $u^{2}=w_{0}^{-1} \bar{u} w_{0} \hat{u}$. In this equation the unreduced length of the right-hand side is $2\left|w_{0}\right|+2|u|$. Any cancellation must start at the juncture $w_{0} \hat{u}$ and successively cancel symbols from $w_{0}$. When $j \leq\left|w_{0}\right|$ symbols have been cancelled, the length of the right-hand side is $2\left|w_{0}\right|+2|u|-2 j$. It follows from this that $w_{0}$ must be cancelled entirely, and when this has occurred the resultant word has unreduced length $2|u|$. Consequently no further cancellation can occur. Moreover, we then have $u \equiv w_{0}^{-1} \bar{u}_{0}$ for some initial segment $\bar{u}_{0}$ of $\bar{u}$. So $\bar{u} \equiv \bar{u}_{0} \bar{u}_{1}$. But then we also have $u=\bar{u}_{1} \hat{u}_{1}$ and so $\bar{u}_{1}=w_{0}^{-1}$ which is impossible unless $w_{0}$ is empty because $w_{0}^{-1} \bar{u} w_{0}$ is freely reduced. So we conclude that if $v_{0}$ is empty then so is $w_{0}$ and $u=\bar{u}=\hat{u}$. But $\hat{u}=v^{-1} u v$ which means that $u=v^{-1} u v$, a contradiction.

In the event that $w_{0}$ is empty a similar argument shows that $v_{0}$ must also be empty and again that $u=\bar{u}=\hat{u}$ and therefore that $u=v^{-1} u v$, a contradiction. So we can assume henceforth that neither $w_{0}$ nor $v_{0}$ is empty.

If $w_{0} v_{0}^{-1}$ is reduced as written, there is no cancellation on the right-hand side of equation (1). Since $u, \bar{u}$ and $\hat{u}$ all have the same length, it follows that $w_{0}$ and $v_{0}$ are empty and we have already dealt with this case.

So we can now suppose there is cancellation at the juncture $w_{0} v_{0}^{-1}$. Since the expressions $w_{0}^{-1} \bar{u} w_{0}$ and $v_{0}^{-1} \hat{u} v_{0}$ are reduced as written, freely reducing the righthand side of equation (1) proceeds by successively cancelling inverse pairs starting from this juncture (there are no others to cancel at any stage). Now the last symbol of $v_{0}$ is the same as the last symbol of $w_{0}$ which is the inverse of the first symbol of $w_{0}^{-1}$. But $u^{2}$ is cyclically reduced as written, so the right-hand side of equation (1) 
must freely reduce to a cyclically reduced word. The only way this can happen is if one of $w_{0}^{-1} \bar{u} w_{0}$ and $v_{0}^{-1} \hat{u} v_{0}$ is entirely cancelled in this process.

First suppose that $w_{0}^{-1} \bar{u} w_{0}$ is entirely cancelled. Then the expression $v_{0}^{-1} \hat{u} v_{0}$ ends in $u^{2}$. There must be $2|u|+4\left|w_{0}\right|$ symbols cancelled, leaving $2|u|$ symbols after the cancellation. Initially there are $2|u|+2\left|w_{0}\right|+2\left|v_{0}\right|$ symbols on the right-hand side of equation (1) so $2|u|+2\left|w_{0}\right|+2\left|v_{0}\right|=4|u|+4\left|w_{0}\right|$ and hence $\left|v_{0}\right|=|u|+\left|w_{0}\right|$. It follows that $v_{0}=\bar{u} w_{0}$ and so

$$
u^{2}=w_{0}^{-1} \bar{u} w_{0} v_{0}^{-1} \hat{u} v_{0}=w_{0}^{-1} \bar{u} w_{0} w_{0}^{-1} \bar{u}^{-1} \hat{u} \bar{u} w_{0}=w_{0}^{-1} \hat{u} \bar{u} w_{0} .
$$

Consider the case in which $\left|w_{0}\right|<|u|$. Since $w_{0}^{-1} \bar{u} w_{0}$ is entirely cancelled, we then have $\hat{u} \equiv w_{0} \hat{u}_{1}$ for some terminal segment $\hat{u}_{1}$ of $\hat{u}$ and $u^{2}=\hat{u}_{1} \bar{u} w_{0}$ where both sides are reduced without cancellation; we emphasise that the notation $\equiv$ is used to express the fact that none of the letters in any of the words cancel in computing the various products. Factoring $\bar{u}$ as $\bar{u}_{0} \bar{u}_{1}$ we can write $\bar{u} \equiv \bar{u}_{0} \bar{u}_{1}$ so that $u \equiv \hat{u}_{1} \bar{u}_{0}$ and $u \equiv \bar{u}_{1} w_{0}$. But from the definition of $\hat{u}_{1}$ it follows that $\left|\bar{u}_{0}\right|=\left|w_{0}\right|$ and so both $\bar{u}_{0}=w_{0}$ and $\hat{u}_{1}=\bar{u}_{1}$. In particular $\bar{u} \equiv w_{0} \bar{u}_{1}$ which contradicts the fact that $w_{0}^{-1} \bar{u} w_{0}$ is reduced without cancellation with $w_{0}$ non-empty. So this case does not arise.

We observe next that $\left|w_{0}\right|$ cannot be greater than or equal to $|u|$, since this implies that both $w_{0}$ and $v_{0}$ end in $u$ and therefore that both $w$ and $v$ end in $u$, contrary to our initial assumption.

Finally, the remaining possibility that $v_{0}^{-1} \hat{u} v_{0}$ is entirely cancelled implies by an essentially identical argument that $u$ and $v$ commute, which is not the case. This completes the proof of the lemma.

Corollary 6. If $r$ and $w$ are elements of a free group which do not commute, then $r^{r^{w}} r^{-2}$ is not conjugate to either $r$ or $r^{-1}$.

Proof. Since $r$ and $w$ do not commute, they freely generate a free group of rank two. In particular then $r$ and $r^{w}$ do not commute. Thus if we set $u=r$ and $v=r^{w}$ then it follows immediately from Lemma 1 that $r^{r^{w}} r^{-2}$ is not conjugate to either $r$ or $r^{-1}$.

3.2. A version of an observation of Graham Higman. We begin the proof of Theorem 1 with the following version of an observation essentially due to Graham Higman.

Lemma 2. Let $J$ be a group generated by two elements $x$ and $y$ of the same finite order m. Suppose that

$$
x^{y}=x^{2}
$$

Then $J=1$. 
Proof. Suppose if possible that $m>1$. Now $y$ conjugates $x$ to its square and so induces an automorphism of $X=\mathrm{gp}(x)$. It follows that $m$ is odd. Let $p$ be the smallest prime dividing $m$. Notice that $p>2$. Let $Z$ be a subgroup of $\operatorname{gp}(x)$ of order $p$, generated say by $z$. Now $y$ conjugates $z$ to its square and hence induces a non-trivial automorphism of $Z$. But the automorphism group of a group of prime order is of order $p-1$. It follows that if $C$ is the centralizer of $z$ in $\operatorname{gp}(y)$ then the order of $\operatorname{gp}(y) / C$ divides $p-1$. It follows that $\operatorname{gp}(y) / C$ contains an element of prime order $q$ dividing $p-1$. But then $q$ divides $m$ and is clearly smaller than $p$. This is a contradiction of the choice of $p$. It follows that $m=1$ and completes the proof of the lemma.

3.3. Checking on the conjugacy of elements in a free group. We will need the following lemma in the proof of Theorem 1.

Lemma 3. Let $F$ be a free group and let $u$ and $v$ be elements of $F$. In addition suppose that $w=w(u, v)$ is product of the elements $u$ and $v$ and their inverses, i.e., $a$ word in $u$ and $v$. If $u=1$ is a consequence of the relation $w(u, v)=1$ and if $w(u, v)=1$ is a consequence of the relation $u=1$, then $u$ is a conjugate of $w$ or $w^{-1}$.

Proof. We denote the normal closure in $F$ of the subset $S$ of $F$ by $\operatorname{gp}_{F}(S)$. Now in order to prove Lemma 3 , let $K=\operatorname{gp}_{F}(w)$ and let $L=\operatorname{gp}_{F}(u)$. Since $w=1$ is a consequence of $u=1$, it follows that $w \in L$ and so $K \leq L$. Moreover the assumption that $u$ is a consequence of $w=1$ implies that $L \leq K$. Thus

$$
K=L
$$

Now in a free group, if $u, w \in F$ and if $\operatorname{gp}_{F}(u)=\operatorname{gp}_{F}(w)$, then Magnus [16] has proved that $u$ is a conjugate of $w$ or a conjugate of $w^{-1}$. This completes the proof of Lemma 3.

3.4. The proof of Theorem 1. Let

$$
G=\langle a, b, \ldots \mid r=1\rangle
$$

be a one-relator group presented on at least two generators. Suppose that $w$ is any element in the ambient free group $F$ on the generators $a, b, \ldots$ which does not commute with $r$ in $F$ and let

$$
G(r, w)=\left\langle a, b, \ldots \mid r^{r^{w}}=r^{2}\right\rangle .
$$

Suppose that $H$ is a finite image of the group $G(r, w)$. Put

$$
r_{i}=w^{-i} r w^{i} \quad(i \in \mathbb{Z}) .
$$


Then the $r_{i}$ are conjugate elements in $G(r, w)$ and consequently have the same order. Moreover

$$
r_{0}^{r_{1}}=r_{0}^{2} .
$$

Consequently by Lemma $3, r_{0}=1$, i.e., $r=1$ in $H$. Therefore $H$ is a finite quotient of $G$.

On the other hand, $G$ is itself a homomorphic image of $G(r, w)$. To see this observe that if we map the generators of $G(r, w)$ to the corresponding generators of $G$, then the image of the relator $r^{r^{w}} r^{-2}$ maps to the identity because the image of $r$ is already 1 in $G$. So every quotient of $G$ is a quotient of $G(r, w)$. In particular all of the finite quotients of $G$ are finite quotients of $G(r, w)$. So we have proved that $G$ and $G(r, w)$ have the same finite images.

We are left with the proof that $G(r, w)$ is not residually finite. Since $r=1$ in every finite quotient of $G(r, w)$, it suffices to prove that $r \neq 1$ in $G(r, w)$. Now if $r=1$ in $G(r, w)$, then $r$ is a consequence of $r^{r^{w}} r^{-2}$. On the other hand, $r^{r^{w}} r^{-2}$ is clearly a consequence of $r$. Then, by Lemma $3, r^{r w} r^{-2}$ is a conjugate of $r$ or $r^{-1}$. However since $r$ and $w$ do not commute in the ambient free group $F$, they freely generate a free group. Consequently, $r$ and $r^{w}$ do not commute and therefore it follows immediately from Lemma 1 that $r^{r^{w}} r^{-2}$ is not a conjugate of $r$ or $r^{-1}$, which contradicts the assumption that $r=1$ in $G$. This completes the proof of Theorem 1 .

It is worth emphasizing that all of the quotients of $G$ are quotients of $G(r, w)$. However, if $G$ is residually finite, $G(r, w)$ is not isomorphic to $G$. It is conceivable, however, that if $G$ is not residually finite, then we have not excluded this possibility. These remarks seem to touch on a possible connection between the isomorphism problem for one-relator groups and the residual finiteness problem.

\section{More on the $G(r, w)$}

The existence of the $G(r, w)$ underlines the increasing awareness of the complexity of one-relator groups. They provide test cases for a number of open problems about one-relator groups. Here are a few sample questions.

- Is the isomorphism problem solvable for the $G(r, w)$ ? (Cf. the solution of the isomorphism problem for the groups $B(p, q)$ by D. I. Moldavanski [19].)

- Can one compute lower bounds for the isoperimetric functions for these groups (cf. S. M. Gersten [10] and A. N. Platonov [21]).

- Are the $G(r, w)$ hopfian?

- Can one use C.F. Miller's family [18] of residually finite groups with unsolvable word problem to show that the isomorphism problem for the $G(r, w)$ is unsolvable? 
- Are the $G(r, w)$ coherent, i.e., are their finitely generated subgroups finitely related?

- Are the groups

$$
G(r, w, n)=\left\langle a, b, \ldots \mid\left(r^{r^{w}} r^{-2}\right)^{n}=1\right\rangle
$$

$n=2,3, \ldots$, residually finite? Virtually free-by-cyclic?

- Much of the discussion about the groups $G(r, w)$ applies also to the more general class of groups

$$
G(r, u, v, w)=\left\langle a, b, \ldots \mid\left(r^{u}\right)^{r^{v}}=\left(r^{w}\right)^{2}\right\rangle .
$$

Whether this remark will turn out to be of interest remains to be seen.

\section{References}

[1] G. Baumslag, Residually finite one-relator groups. Bull. Amer. Math. Soc. 73 (1967), 618-620. Zbl 0153.34904 MR 0212078

[2] G. Baumslag, A non-cyclic one-relator group all of whose finite quotients are cyclic. $J$. Austral. Math. Soc. 10 (1969), 497-498. Zbl 0214.27402 MR 0254127

[3] G. Baumslag, Finitely generated cyclic extensions of free groups are residually finite. Bull. Austral. Math. Soc. 5 (1971), 87-94. Zbl 0216.08301 MR 0311776

[4] G. Baumslag, Some open problems. In Summer school in group theory in Banff, 1996, CRM Proc. Lecture Notes 17, Amer. Math. Soc., Providence, RI, 1999, 1-9. Zbl 01284206 MR 1653682

[5] B. Baumslag and F. Levin, A class of one relator groups with torsion. Arch. Math. (Basel) 33 (1979), 209-215. Zbl 0411.20020 MR 559539

[6] G. Baumslag and D. Solitar, Some two-generator one-relator non-Hopfian groups. Bull. Amer. Math. Soc. 68 (1962), 199-201. Zbl 0108.02702 MR 0142635

[7] J. L. Dyer, Separating conjugates in amalgamated free products and HNN extensions. $J$. Austral. Math. Soc. Ser. A 29 (1980), 35-51. Zbl 0429.20033 MR 566274

[8] V. Egorov, Residual finiteness of some groups with a single defining relation (Russian). In Algebraicheskie sistemy, Ivanov. Gos. Univ., Ivanovo 1981, 100-121. Zbl 0524.20018 MR 0745301

[9] M. Feighn and M. Handel, Mapping tori of free group automorphisms are coherent. Ann. of Math. (2) 149 (1999), 1061-1077. Zbl 0938.20022 MR 1709311

[10] S. M. Gersten, Isodiametric and isoperimetric inequalities in group extensions. Preprint, University of Utah, Salt Lake City 1991.

[11] A. Juhász, Small cancellation theory with a unified small cancellation condition I. $J$. London Math. Soc. (2) 40 (1989), 57-80. Zbl 0647.20030 MR 1028914 
[12] A. Juhász, Solution of the conjugacy problem in one-relator groups. In Algorithms and classification in combinatorial group theory (Berkeley, CA, 1989), Math. Sci. Res. Inst. Publ. 23, Springer, New York 1992, 69-81. Zbl 0749.20019 MR 1230629

[13] A. Juhász, Some applications of small cancellation theory to one-relator groups and one-relator products. In Geometric group theory (Sussex, 1991), Vol. 1, London Math. Soc. Lecture Note Ser. 181, Cambridge University Press, Cambridge 1993, 132-137. Zbl 0807.20031 MR 1238521

[14] A. Karrass, W. Magnus, and D. Solitar, Elements of finite order in groups with a single defining relation. Comm. Pure Appl. Math. 13 (1960), 57-66. Zbl 0091.02403 MR 0124384

[15] W. Magnus, Über diskontinuierliche Gruppen mit einer definierenden Relation (Der Freiheitssatz). J. Reine Angew. Math. 163 (1930), 141-165. JFM 56.0134.03

[16] W. Magnus, Das Identitätsproblem für Gruppen mit einer definierenden Relation. Math. Ann. 106 (1932), 295-307. JFM 58.0125.01 Zbl 0004.09709 MR 1512760

[17] J. P. McCammond and D. T. Wise, Coherence, local quasiconvexity, and the perimeter of 2-complexes. Geom. Funct. Anal. 15 (4) (2005), 859-927. MR 2221153

[18] C. F. Miller, III, On group-theoretic decision problems and their classification. Ann. of Math. Stud. 68, Princeton University Press, Princeton, NJ, 1971. Zbl 0277.20054 MR 0310044

[19] D. I. Moldavanskiı̌, Isomorphism of the Baumslag-Solitar groups. Ukraïn. Mat. Zh. 43 (1991), 1684-1686; English transl. Ukrainian Math. J. 43 (1991), 1569-1571. Zbl 0782.20027 MR 1172309

[20] B. B. Newman, Some results on one-relator groups. Bull. Amer. Math. Soc. 74 (1968), 568-571. Zbl 0174.04603 MR 0222152

[21] A. N. Platonov, An isoparametric function of the Baumslag-Gersten group. Vestnik Moskov. Univ. Ser. I Mat. Mekh. 2004 (3) (2004), 12-17; English transl. Moscow Univ. Math. Bull. 59 (3) (2004), 12-17. Zbl 1084.20022 MR 2127449

[22] S. J. Pride, The isomorphism problem for two-generator one-relator groups with torsion is solvable. Trans. Amer. Math. Soc. 227 (1977), 109-139. Zbl 0356.20037 MR 0430085

[23] Z. Sela, The isomorphism problem for hyperbolic groups I. Ann. of Math. (2) 141 (1995), 217-283. Zbl 0868.57005 MR 1324134

[24] Wise, D. Residual finiteness of positive one-relator groups. Comment. Math. Helv., to appear.

[25] Wise, D. The residual finiteness of one-relator groups with torsion. In preparation.

Received August 10, 2006; revised March 13, 2007

G. Baumslag, Department of Mathematics and Computer Science, City College of New

York, Convent Ave. and 138th Street, New York, N.Y. 10031, U.S.A.

E-mail: gilbert@groups.sci.ccny.cuny.edu 
C. F. Miller III, Department of Mathematics and Statistics, University of Melbourne, Parkville 3052,

Australia

E-mail: c.miller@ms.unimelb.edu.au

D. Troeger, Department of Computer Science, City College of New York, Convent Ave. and 138th Street, New York, N.Y. 10031, U.S.A.

E-mail: dtroeger@cs.ccny.cuny.edu 